\section{Aspectos Epidemiológicos das Relações entre Tremor Essencial (TE) e Doença de Parkinson (DP)}

\section{RESUMO}

Objetiva-se a revisão da literatura acerca dos aspectos epidemiológicos das relações entre Tremor Essencial e doença de Parkinson. Analisam-se dois aspectos: risco de pacientes com Tremor Essencial desenvolverem doença de Parkinson e freqüência de Tremor Essencial em familiares de pacientes com doença de Parkinson. Observa-se grande variaçẫo nas taxas de associação entre as patologias, oscilando de 0 a $20 \%$. Essas discrepâncias possivelmente relacionam-se às heterogeneidades metodológicas não permitindo conclusões definitivas. No entanto, alguns estudos atentam para a possivel associação do Tremor Essencial com um subgrupo de pacientes com doença de Parkinson, que inauguram o quadro clínico da doença com tremor, mínimo componente rígido acinético e evoluem lenta $e$ progressivamente. Ainda através desses estudos, não há dados que sustentem associação genética entre ambas as condições.

\section{UNITERMOS}

Tremor essencial, doença de Parkinson.
- Doutor em Neurologia. Chefe do Ambulatório de Desordens do Movimento do Departamento de Neurologia do Hospital das Clínicas da FMUSP.

** Mestre em Neurologia. Médica Assistente do Serviço de Neurologia de Emergência do Hospital das Clínicas da FMUSP.

\section{INTRODUÇÃO}

Apesar da observação de James Parkinson, em $1817^{20}$, de que a paralisia agitante e o TE eram entidades distintas, a possibilidade de que uma relação etiológica entre as duas condições possa existir tem sido debatida ao longo dos anos. Gowers, em $1888^{7}$, foi um dos primeiros a notar a possível relação entre as duas patologias, observando alguns casos com caráter intermediário entre as duas afecções. Minor, em $1922^{4}$, sugeriu que o TE fosse uma forma frustrada de DP, ou que as duas desordens representassem extremos de um contínuo. Para outros autores as duas condições podem ocorrer em associação em um mesmo paciente com frequiência suficiente para sugerir que ambas possam estar relacionadas, quer patológica ou geneticamente ${ }^{1,5,8}$.

Um dos aspectos das relações entre essas duas patologias é a possibilidade de que pacientes com TE ou seus familiares tenham uma predisposição genética para o desenvolvimento de DP. Se esse conceito fosse correto, as seguintes observações deveriam ser esperadas: casos "complicados" de TE com sinais adicionais, isto é, sinais de parkinsonismo; freqüência aumentada de DP em pacientes com TE; freqüência aumentada de TE em famílias de pacientes com DP e frequiência aumentada de DP em famílias de pacientes com TE.

A presença de sinais de parkinsonismo em pacientes com TE permanece uma questão controvertida ${ }^{7}$. A presença desses sinais em pacientes com TE pode indicar: evolução natural do TE; coexistência incidental de síndrome parkinsoniana ou DP idiopática; risco exagerado de síndrome parkinsoniana ou DP idiopática; ou ainda, existência de forma não-usual de DP progressiva onde predomina o tremor ${ }^{22}$.

Quando um indivíduo apresenta TE associado à DP, a sequiência usual é o aparecimento inicial do TE e após, sinais da DP. Considerando-se esse padrão de evolução, há um debate ativo sobre o risco de parkinsonismo em TE. Adicionalmente, discute-se o risco de familiares de pacientes com TE desenvolverem DP, e a incidência de TE em famílias de pacientes com DP $\mathrm{P}^{11,15,23}$.

A comprovação de correlação entre as duas condições seria importante na medida em que influenciaria a terapia com drogas visando ao tratamento 
neuroprotetor, abordagem do seguimento clínico de pacientes com TE e prognóstico dessa doença ${ }^{10}$.

Além disso, os estudos procurando demonstrar correlação entre as duas patologias levam à busca de um marcador biológico ou genético visando à distinção entre o TE e a DP.

Neste artigo revisamos a literatura a respeito dos principais estudos epidemiológicos na pesquisa de associação entre as duas patologias. Uma visão geral destes pode ser apreciada na tabela 1 .

Uma correlação fortemente positiva entre TE e DP tem sido sugerida por vários estudos como os de: Hornabrook \& Nagurney ${ }^{8}$, Barbeau \& Pourcher ${ }^{1}$, Geraghty et al. ${ }^{7}$, Lou \& Jankovic ${ }^{13}$, Koller et al ${ }^{10}$.

Em estudo populacional de pacientes com TE em Papua, Nova Guiné, Hornabrook \& Nagurney ${ }^{8}$ reportaram três casos de DP entre 175 pacientes com TE. Os autores através de projeções estatísticas observaram que esses resultados refletiam naquela população-risco 35 vezes maior de DP em pacientes com TE. Contudo, esse estudo tem sido criticado por apresentar erros metodológicos tais como: pequeno número de pacientes na amostra e critérios diagnósticos para TE mal-definidos ${ }^{19}$.

Barbeau \& Pourcher ${ }^{1}$ observaram casos de TE entre pacientes que apresentavam DP de início precoce. Os

\section{TABELA 1}

Aspectos epidemiológicos da associação entre Tremor Essencial e Doença de Parkinson

\begin{tabular}{lccc}
\hline $\begin{array}{l}\text { Autor } \\
\text { Hornabrook; }\end{array}$ & Ano & Tipo de Estudo & Observação \\
$\begin{array}{l}\text { Nagurney } \\
\text { Barbeau; } \\
\text { Pourcher }\end{array}$ & 1982 & Pesquisa Populacional & Associação positiva \\
\hline
\end{tabular}

\begin{tabular}{lccc}
\hline Rajput et al. & 1984 & Revisão de registros médicos & Nenhuma associação \\
\hline Geraghty et al. & 1985 & Série clínica & Associação positiva \\
\hline Martinelli et al. & 1987 & Série clínica & Nenhuma associação \\
\hline Barucha et al. & 1988 & Série clínica & Nenhuma associação \\
\hline Cleeves et al. & 1988 & Série clínica & Nenhuma associação \\
\hline Lou; Jankovic & 1991 & Série clínica & Associação positiva \\
\hline Bain et al. & 1994 & Série clínica & Nenhuma associação \\
\hline Koller et al. & 1994 & Série clínica & Associação positiva
\end{tabular}

autores referiram-se a esses pacientes como "TE relacionado a parkinsonismo", sugerindo que existe uma variante de TE relacionado à $\mathrm{DP}$, ou que poderia haver um "fator gatilho", ainda não conhecido, que levaria ao desenvolvimento de DP em pacientes com TE.

Geraghty et al. ${ }^{7}$ observaram que $19 \%$ de 130 pacientes com TE examinados apresentavam associação entre TE e DP. De acordo com esses autores, através de projeções de estudos epidemiológicos, esperar-se-ia nesse estudo que somente um paciente apresentasse concomitância entre TE e DP. Entretanto, observaram que a DP foi 24 vezes mais freqüente em pacientes com TE acima de 60 anos de idade do que o esperado na população geral. Se pacientes com TE de todas as idades fossem incluídos, a chance de desenvolvimento de DP era 19 vezes maior que da população geral. Constataram ainda que a frequiência de DP no subgrupo de pacientes com TE, com evolução maior que cinco anos, era 18 vezes maior do que a população geral. Outro aspecto interessante observado nesse estudo foi que o início do parkinsonismo era mais tardio nos pacientes com associação entre TE e DP do que em pacientes com DP isolada. Esses autores compararam esses pacientes com associação das patologias (TE/DP) com um grupo de pacientes com DP. Essa análise levou à observação de que bradicinesia, instabilidade postural e dificuldade à marcha eram mais severas nesse último grupo, enquanto que rigidez, tremor e incapacidade funcional apresentavam-se em mesma intensidade nos dois grupos.

Em uma análise retrospectiva de 350 pacientes em uma clínica de desordens do movimento, Lou \& Jankovic ${ }^{13}$ observaram que a frequiência de DP associada a TE era de $20 \%$. Os autores igualmente admitem que alguns pacientes com TE podem ter risco aumentado para desenvolver DP. Lang et al. ${ }^{12}$ consideram que a alta freqüência de associação entre TE e DP observada nesse estudo seria por erros metodológicos. Salientaram que por um lado aqueles autores rotularam todos os tremores posturais como TE, o que pode ter levado à inclusão de alguns casos de tremor postural próprio da DP, e por outro a amostra de pacientes era originária de uma clínica específica de desordem do movimento. Este último fato pode ter levado à seleção de pacientes de acordo com os autores.

Um estudo com grande casuística (678 pacientes com TE) foi realizado por 


\section{TABELA 2}

Aspectos epidemiológicos da freqüência de Tremor Essencial em familiares de pacientes com Doença de Parkinson

\begin{tabular}{lccc}
\hline Autor & Ano & Tipo de Estudo & Observação \\
\hline Duvoisin et al. & 1969 & Série clínica & Nenhuma associação \\
\hline Martin et al. & 1973 & Série clínica & Associação positiva \\
\hline Martilla; Rinne & 1976 & Série clínica & Nenhuma associação \\
\hline $\begin{array}{l}\text { Barbeau; } \\
\text { Pourcher }\end{array}$ & 1982 & Série clínica & Associação positiva \\
\hline Roy et al. & 1983 & Série clínica & Associação positiva \\
\hline $\begin{array}{l}\text { Martilla et al. } \\
\text { Lang et al. }\end{array}$ & 1984 & Série clínica & Nenhuma associação \\
\hline Cleeves et al. & 1986 & Série clínica & Associação positiva \\
\hline Jankovic et al. & 1995 & Série clínica & Nenhuma associação \\
\hline
\end{tabular}

mesmo estudo, não havia frequiência aumentada de história familiar de DP em pacientes com TE quando os mesmos foram comparados com os grupos-controles.

Lang et al. ${ }^{11}$ observaram dados semelhantes. Constataram história familiar positiva de TE em 17\% dos pacientes com DP, enquanto que nos indivíduos-controles essa freqüência era de $5,6 \%$. No grupo de pacientes com história familiar positiva para TE, apenas $3,8 \%$ apresentavam padrão de transmissão autossômica dominante.

Jankovic et al. ${ }^{9}$ relataram maior incidência de TE em familiares de pacientes com DP do que em parentes de portadores de paralisia supranuclear progressiva e indivíduos-controles e, com base nesses dados, admitem que possa haver uma associação entre TE e DP.

Contudo, outros estudos não têm comprovado o aumento da incidência de

Koller et al. ${ }^{10}$, que coletaram dados de uma clínica de desordens do movimento e de consultórios de neurologistas. Obtiveram uma freqüência de DP em pacientes com TE de aproximadamente $6 \%$. Atribuem essa taxa de associação mais baixa quando comparada com estudos anteriores à metodologia empregada, já que sendo incluídos pacientes atendidos em consultórios neurológicos, minimizou-se a distorção da seleção de pacientes.

Por outro lado os estudos de Rajput et al. ${ }^{21}$, Martinelli et al. ${ }^{18}$, Barucha et al. ${ }^{3}$, Cleeves et al. ${ }^{4}$, Bain et al. ${ }^{2}$ mostraram taxas de associação entre TE e DP variando de 0 a $3 \%$, indicando que pacientes com TE não apresentam risco aumentado para desenvolver DP.

Outra linha de pesquisa epidemiológica tem estudado a frequiência do TE em familiares de pacientes com DP. Um resumo de alguns estudos podem ser observados na tabela 2 .

Martin et al. ${ }^{14}$ relataram presença de TE em torno de $15 \%$ dos familiares de pacientes com DP estudados.

Barbeau \& Pourcher ${ }^{1}$ observaram através de análise retrospectiva que $20 \%$ dos pacientes com DP, cuja doença havia começado com tremor, e $8 \%$ dos que apresentavam rigidez e/ou acinesia como sintoma inicial, tinham história familiar de TE.

Roy et al. ${ }^{23}$ conduziram um estudo prospectivo de pacientes com DP observando que aqueles em que a doença se iniciava precocemente apresentavam maior freqüência de história familiar de TE. Entretanto, nesse
TE em parentes de pacientes com DP. Assim, Duvoisin et al. ${ }^{6}$ examinaram todos os membros das famílias de seus pacientes com DP e constataram a mesma frequiência de TE tanto em irmãos de pacientes com DP quanto nas esposas e familiares dos mesmos.

Martilla \& Rinne ${ }^{15}$ observaram que $5,8 \%$ dos familiares de pacientes com DP apresentavam TE, ao passo que essa taxa foi de $8,1 \%$ nos indivíduoscontroles. Deve-se ressaltar que essa alta taxa de TE em familiares de indivíduos-controles era esperada dada a alta prevalência dessa condição na população estudada (Finlândia).

Martilla et al. ${ }^{17}$ não evidenciaram aumento de freqüência de DP em familiares de pacientes com TE quando esses foram comparados a um grupo-controle. Esses autores sugeriram que esses indivíduos têm o mesmo risco da população geral de desenvolver DP.

Da mesma forma, Cleeves et al. $^{4}$, avaliando a incidência de TE em parentes de primeiro grau de pacientes com DP. não observaram diferença com relação ao grupo-controle.

Martilla \& Rinne ${ }^{16}$ analisaram a ocorrência de TE e DP em familiares de pacientes com DP de início precoce. Esses autores observaram que a incidência de DP e TE nesses indivíduos não era significativamente diferente da população geral. Concluíram que esses dados não confirmam algum padrão genético em DP de início precoce ou associação de TE e DP. 


\section{CONSIDERAÇÕES FINAIS}

Os estudos epidemiológicos de avaliação da prevalência do TE mostram claramente que essa condição é bastante comum em indivíduos acima de 40 anos, sendo considerada a desordem do movimento mais comum. Por outro lado, admite-se que a incidência de DP aumenta após os sessenta anos de idade na população geral. Dessa forma, pode-se admitir que o TE e a DP ocorram coincidentemente em indivíduos mais idosos.

Nos estudos epidemiológicos analisados observa-se grande variação nas taxas de associação entre as duas patologias, as quais oscilaram de 0 a $20 \%$. Essas discrepâncias possivelmente estão relacionadas a diferenças metodológicas e características das populações estudadas. As principais distorções metodológicas são: curto período de seguimento dos pacientes, variabilidade numérica das amostras de pacientes com TE e as dificuldades diagnósticas entre TE e DP. Além disso, não há especificação das formas de DP (com predomínio de tremor ou quadro rígidoacinético) que estão sendo consideradas.

Embora não se possa concluir por uma associação entre TE e DP através da análise desses estudos epidemiológicos, alguns destes chamam a atenção para a possível associação de TE com um subgrupo de pacientes com DP. Esse subgrupo incluiria aqueles pacientes que inauguram o quadro clínico da doença com tremor e mínimo componente rígido-acinético e apresentam evolução relativamente lenta e benigna.

Os resultados dos estudos avaliando a frequiência de TE em familiares de pacientes com DP são igualmente discrepantes, porém de maneira geral não há dados que sustentem associação genética entre ambas as condições.

Por esses motivos, o ideal seria um estudo longitudinal de longo prazo visando avaliar de forma adequada se os portadores de TE realmente apresentam risco de desenvolver DP.

\section{SUMMARY}

The epidemiological relationship between essential tremor and Parkinson's disease.

The epidemiological clues in the literature to a possible relationship between essential tremor and Parkinson's disease are reviewed. The risk of patients with essential tremor to develop Parkinson's disease (PD) and the frequency of essential tremor in family members of PD patients are the two main points in the review. A large variation in this association, ranging from 0 to $20 \%$ is observed. The discrepancies are possibly related to heterogenous methodology, thus allowing no definitive conclusions. However, a PD subgroup in whom the disease start with tremor, minimal rigidity and bradykinesia with a slow progression may show some association with essential tremor, according to some studies. The available data do not support a genetic link between the two conditions.

\section{KEYWORDS}

Essential tremor, Parkinson's disease.

\section{Referências}

1. Barbeau, A.; Poucher, E. New data on the genetics parkinson disease. Can. J. N. Sci., 9: 53-60, 1982.

2. Bain, P.G.; Findley, L.J.; Thompson, P.D.; Gresty, M.A.; Rothwell, J.C.; Harding, A.E.; Marsden, C.D. A study of hereditary essential tremor. Brain, 117: 805-24, 1994.

3. Bharucha, N.E.; Bharucha, E.P.; Bharucha, A.E.; Bhise, V.A.; Schoenberg, B.S. Prevalence of essential tremor in the Parsi Community of Bombay, India. Arch. Neurol., 44: 907-8, 1988.

4. Cleeves, L.; Findley, L.J.; Koller, W. Lack of association between essential tremor and parkinson disease. Ann. Neurol., 24: 23-6, 1988.

5. Critchley, M. Observations on essential (heredofamilial) tremor. Brain, 72: 113-39, 1949.

6. Duvoisin, R.C.; Grearing, F.R.; Schweitzer, M.B.; Yahr, M.D. A family study of parkinsonism. In: Barbeau, A. \& Brunette, J.R. eds. Progress in Neurogenetics. Amsterdan, Excerpta Medica, p. 492-6, 1969.

7. Geraghty, J.J.; Jankovic, J.; Zetusky, W.J. Association between familial tremor and parkinson disease. Ann. Neurol., 19: $306-$ 7, 1985.

8. Hornabrook, R.W.; Nagurney, J.T. Essential tremor in Papua New Guinea. Brain, 99: 113-39, 1976.

9. Jankovic, J.; Beach, J.; Schwartz, K.; Contant, C. Tremor and longenvity in relatives of patients with parkinson's disease, essential tremor, and control subjects. Neurology, 45: 645-8, 1995.

10. Koller, W.C.; Busenbark, K.; Miner, K. The relationship of essential tremor to other movement disorders: report on 678 patients. Ann. Neurol., 35: 711-23, 1994a.

11. Lang, A.E.; Kierans, R.N.; Blair, R.D.G. Assoaciation between familial tremor and parkinson's disease. Ann. Neurol., 19:3067, 1986.

12. Lang, A.; Quinn, N.; Marsden, C.D. Essential tremor [letter]. Neurology, 42: 1432-33, 1992.

13. Lou, S.J.; Jankovic, J. Essential tremor clinical correlates in 350 patients. Neurology, 41: 234-8, 1991.

14. Martin, W.E.; Young, W.I.; Anderson, V.E. Parkinson's disease: a genetic study. Brain, 96: 495-506, 1973.

15. Martilla, R.J.; Rinne, U.K. Arteriosclerosis, heredity and some previous infections in the etiology of Parkinson's disease: a case control study. Clin. Neurol. Neurosurg., 79: 45-56, 1976.

16. Martilla, R.J.; Rinne, U.K. Parkinson's disease and essential tremor in families of patients with early-onset parkinson's disease. J. Neurol. Neurosurg. Psychiatry, 51: 429-31, 1988.

17. Martilla, R.J.; Rautakorpi, I.; Rinne, U.K. The relation of essential tremor to Parkinson's disease. J. Neurol. Neurosurg. Psychiatry, 47: 734-5, 1984.

18. Martinelli, P.; Gabellini, A.S.; Gulli, M.R.; Lugaresi, E. Different clinical features of essential tremor: a 200 patient study. Acta Neurol. Scand., 75: 106-11, 1987.

19. Pahwa, R.; Koller, W. Is there a relationship between parkinson's disease and essential tremor. Clin. Neuropharmacol., 16: 30-5, 1993.

20. Parkinson, J. An essay on the shaking palsy. London, Whittingham \& Rowland, 1817.

21. Rajput, A.H.; Offord, K.P.; Beard, C.M.; Kurland, L.T. Essential tremor in Rochester, Minesota: a 45 year study. J. Neurol. Neurosurg. Psychiatry, 47: 466-70, 1984.

22. Rajput, A.H.; Rozdilsky, B.; Ang, L.; Rajput, A. Significance of parkinsonian manifestations in essential tremor. Can. J. Neurol. Sci., 20: 114-7, 1993.

23. Roy, M.; Boyer, L.; Barbeau, A. A prospective study of 50 cases of familial Parkinson's disease. Can. J. Neurol Sci., 10: 37-42, 1983. 